\title{
Surgical treatment of previously embolized craniocervical junction dural arteriovenous fistula
}

\author{
Thomas J. Sorenson, BS, ${ }^{1,2}$ Lucio De Maria, MD, ${ }^{1}$ Leonardo Rangel Castilla, MD,, \\ and Giuseppe Lanzino, MD ${ }^{1,3}$
}

Departments of ${ }^{1}$ Neurologic Surgery and ${ }^{3}$ Radiology, Mayo Clinic; and ${ }^{2}$ School of Medicine, University of Minnesota, Minneapolis, Minnesota

Craniocervical junction dural arteriovenous fistulas (dAVFs) are rare vascular lesions with a potentially dangerous natural history due to the onset of neurological deficit secondary to intracranial hemorrhage or myelopathy due to venous congestion. Despite advances in endovascular techniques, many dAVFs located in this area continue to require surgical treatment as embolization is often not feasible or safe. In this video, the authors illustrate a patient with a symptomatic craniocervical junction dAVF who had undergone attempted Onyx embolization at another institution. Because of persistent filling of the fistula and worsening myelopathy after the previous attempt, the patient was referred to the authors' clinic for definitive surgical treatment. The video illustrates the typical location of the early draining vein in most craniocervical junction dAVFs immediately below the emergence of the vertebral artery from the dura. The patient underwent successful definitive clip ligation of the fistula, which was exposed through a lateral suboccipital craniotomy.

The video can be found here: https://youtu.be/Bvg6VKLgwO0.

KEYWORDS dural arteriovenous fistula; clipping; microsurgery; embolization; Onyx; video 